Journal of Alloys and Compounds, Volume 859, April 2021, Article number 157864

DOI: $10.1016 /$ j.jallcom.2020.157864

\title{
Investigation on the mechanically-induced nanocrystallization in metallic glasses
}

\author{
C. Ammari², M. A. Yousfi ${ }^{1,2}$, K. Hajlaoui ${ }^{2,3}$, K. Georgarakis ${ }^{4}$ \\ ${ }^{1}$ Department of Mechanical Engineering, College of Engineering King Khalid Univerity, Abha KSA. \\ ${ }^{2}$ Mechnaical Laboratory of Sousse LMS, ENISo, University of Sousse, Tunisia, \\ ${ }^{3}$ Al Imam Mohammad Ibn Saud Islamic University (IMSIU), P.O. Box 5701, Riyadh 11432, Saudi Arabia \\ ${ }^{4}$ School of Aerospace, Transport and Manufacturing, Cranfield University, MK43 0AL, UK.
}

\begin{abstract}
Shear-induced nanocrystallization in bent ribbons of $\mathrm{Pd}_{40} \mathrm{Cu}_{30} \mathrm{Ni}_{10} \mathrm{P}_{20}$ metallic glass has been quantitatively investigated via synchrotron radiation. The formed nanocrystals volume fraction during deformation has been directly estimated from X-ray diffraction spectra using peaks area integration. The nanocrystallization process during deformation was found to be strongly linked with the microstructure configuration of shear bands in amorphous alloys. A constitutive model based on free volume approach has been introduced to describe the kinetic of mechanically induced nanocrystallization. The solution of the coupled constitutive equations of the model, fitted to experimental data, permits to determine the physical and mechanical parameters governing the phenomena of shear-induced crystallization in metallic glasses.
\end{abstract}

Keywords: Metallic glass, Deformation-induced nanocrystallization, Crystal volume fraction, Free volume, Activation energy. 


\section{Introduction}

Metallic glasses (MGs) are attractive and promising new structural materials. The absence of long range atomic order, lattice and crystal defects provides them a unique service performances, including high mechanical strength, high hardness, large elastic strain limit, high wear and corrosion resistance and low magnetic energy loss [1,2]. However, at ambient temperature, MGs exhibit poor ductility. Unfortunately, they tend to break after yielding leading to a catastrophic failure. The poor plastic deformation results from highly localized shear banding as well as shear softening [3-5]. The catastrophic brittle deformation becomes the major weakness for wide structural application of MGs as engineering materials [6-8]. To circumvent the problem of room-temperature brittleness, several post treatment methods have been introduced including surface mechanical treatment [9], defect printing [10], ion irradiation [11], notching [12], and ultrasonic excitation [13]. Other attempts aimed to hinder localized shear band formation by ex-situ mixing or in-situ generating a micro/nano-scale crystallites embedded in amorphous matrixes to produce a composite structure, consisting of a crystalline phase embedded in an amorphous matrix [14-17]. Numerous experimental data showed an improvement of the material's properties due to the effect of the introduced second phase [1821]. It was found that nanocrystals act as an arrest barriers to shear band propagation, improving thus the global ductility. The size, volume fraction, and microstructural transformation of the second phase have proven critical in optimizing the ductility of these materials. Recently, there has been considerable interest in the shear induced nanocrystallization and structural transformation in MGs which provide a novel way for further improving ductility compared to monolithic bulk-MGs [22-25]. This phenomenon was detected first by Chen et al [26] in bent ribbons of Al-based metallic glass. Latterly, other studies have suggested that ambienttemperature mechanical deformation can induce crystallization achieved by rolling [27], ball milling [28], high-pressure torsion [29], nanoindentation [30] and uniaxial compression [31]. 
Therefore, the understanding of crystallization mechanism/kinetics in metallic glass systems during deformation is essential for the preparation, synthesizing and engineering application of metallic glasses in various scientific fields. Previous transmission electron microscopy (TEM) experiments study in deformed metallic glasses have largely been limited to qualitative imaging studies with high enough resolution to examine shear bands [14,15,22,23], but have difficulties in quantitative interpretations. Recent advancements in XRD techniques have, however, allowed the observation of the evolution of locally resolved atomic short and medium range order, with nanometer resolution [32,33], providing much more valuable quantitative information on crystallization processes of metallic glass materials during in-situ deformation. To quantitatively highlight this issue, metallic glass ribbons of $\mathrm{Pd}_{40} \mathrm{Cu}_{30} \mathrm{Ni}_{10} \mathrm{P}_{20}$ were deformed at room temperature by means of bending test. X-ray diffraction using synchrotron radiation is used to map out the structure changes in the Pd-based glassy ribbon and quantitatively analyze the shear induced nanocrystallization mechanism. This work deal with the results from the same experiment as in the previous investigation [33]. A micro-mechanical model is employed as well to investigate the evolution of the nanoparticles volume fraction during the deformation route of metallic glasses.

\section{Experimental and results}

$\mathrm{Pd}_{40} \mathrm{Cu}_{30} \mathrm{Ni}_{10} \mathrm{P}_{20}$ (in at \%) alloy ingots were achieved by arc melting the high purity alloy components under Ti-purified Ar atmosphere. Rapidly solidified ribbon specimens were prepared by re-melting an appropriate amount of the ingot in quartz crucible by induction heater and ejecting through a $0.5 \mathrm{~mm}$-diameter circular nozzle onto a copper wheel rotating with a surface speed of 1000 to $2000 \mathrm{rpm}$. The resulting specimens have a thickness of $30-70 \mu \mathrm{m}$ depending on wheel rotation speed. The specimens were then strained by bending them to a curvature radius $\mathrm{R}$ such that it generates plastic deformation in the specimen. The morphology of side surfaces after bending deformation are shown in SEM image of figure 1 (JEOL JSM- 
6400). Extrados and intrados sustain purely tension and compression, respectively. Consider a flat ribbon of metallic glass of thickness $d$. If this ribbon is bent to a curvature radius $R$, the strain on the tension (or compressive) side of the bent specimen can be approximated by [34, 35]:

$\varepsilon=\mathrm{y} / \mathrm{R}$

where $\mathrm{y}$ is the distance from the neutral axis (see figure $1(\mathrm{~b})$ ). For this strain to be over the elastic limit (i.e. $\varepsilon_{\text {ela }} \sim 2 \%$ ), the radius of curvature $\mathrm{R}$ must be in the range of $\mathrm{R}<\mathrm{y} / \varepsilon_{\mathrm{ela}} \approx 50 \mathrm{y}$. For metallic glass ribbons with thickness $35 \mu \mathrm{m}$, a curvature bending radius of approximately $30 \mu \mathrm{m}$ is used. This curvature corresponds to maximum stain of $58 \%$ at the edge of the ribbon. The strain may reach up to the estimated values because of its local character and the bending confinements. The strained zones in the cross section along the width of the bent ribbon was scanned by X-ray in transmission at the ESRF ID11 beamline [36]. A focused $92.5 \mathrm{KeV}$ photon energy corresponding to an X-ray wave length of $0.0134 \mathrm{~nm}$ was used with a spot size of $2 \mathrm{x} 2$ $\mu \mathrm{m}$. The diffracted intensity in transmission was recorded by a 2-d CCD camera placed perpendicular to the incident beam away from the specimen of about $1 \mathrm{~mm}$. Figure 2 presents a scanning electron microscope (SEM) image together with a representative CCD screen images of the microprofiling in transmission taken in both sides of the neutral axis. Very fine rings (see arrows) due to nanometer-scale crystallites (of the order of 5-10 $\mathrm{nm}$ [37]) formation during deformation is seen only in the compressed part of the ribbon thickness. The shear-induced nanocrystallization in analogous complex stress states has been also reported in many previous published works $[30,38-40]$ and was attributed to the role of the hydrostatic component of the stress and the free volume distribution. Radial integration of these concentric rings yields to the XRD profile changes along the width of the ribbon. Figure 3 shows typical diffraction patterns 
of $\mathrm{Pd}_{40} \mathrm{Cu}_{30} \mathrm{Ni}_{10} \mathrm{P}_{20}$ amorphous alloy corresponding to a series of successive diffraction profile recorded along the width of the ribbon. In going from the upper part $\left(\mathrm{D}_{0}\right)$ towards the extremity of ribbon $\left(D_{20}\right)$, qualitative and quantitative changes are revealed from the superposition of diffracted profiles. Nanocrystals appear with peak positions correspond to $\mathrm{Cu}_{3} \mathrm{Pd}$ precipitates as reported during thermal crystallization of $\mathrm{Pd}-\mathrm{Cu}-\mathrm{Ni}-\mathrm{P}$ Alloys [41]. Diffraction patterns were obtained every approximately $4 \mu \mathrm{m}$ incremental displacement during scanning of strained zones. The position of the beam spot is denoted by $\mathrm{D}_{0}, \mathrm{D}_{4}$ (in tensile side), $\mathrm{D}_{8}$ (neutral axis), $\mathrm{D}_{12}, \mathrm{D}_{16}, \mathrm{D}_{20}$ (in compression side) as shown in figure 2.

\section{Measurement of crystals volume fraction during deformation}

Structural analysis reveals that deformation induced partial nanocrystallization with kinetics that depend on the extent of plastic deformation. To our knowledge, no quantitative measurement of the crystallization process during deformation is available. Quantification of crystal volume fraction evolution during straining can be determined from the changes in diffraction patterns shown in figure 3. The integrated areas of the amorphous and crystalline peaks could be used to calculate the crystal volume fraction according to the equation $[42,43]$ :

$\emptyset_{\mathrm{S}}=\frac{\mathrm{A}_{\mathrm{c}}}{\mathrm{Ac}+\mathrm{Aa}}$

Where $\theta_{\mathrm{s}}$ is the volume fraction of precipitated nanocrystals, $A_{a}$ is the total integrated area corresponding to the amorphous phase and $\mathrm{A}_{\mathrm{c}}$ is the total integrated area corresponding to the crystalline phase as illustrated in figure 4 (a). These results of crystal volume fraction versus deformation are then obtained from equation 1 and 2 and are presented in the figure 4(b). Nanocrystallization took place after specimen was deformed for $\sim 13 \%$, which is close to 
previous works where they showed that the crystalline phases may congregate at dominant shear bands after the macroscopic strain reached over 5-20\% [44-45]. Obviously, the nanocrystallization is a function of deformation within the shear bands and the fraction of the nanocrystalline phase increases with shear strains and deformation time.

\section{4- Discussions and modelling approach}

The microscopic mechanisms of strain-induced nanocrystallization in metallic glasses are of significant fundamental interest and have yet to be conclusively explained. A number of suggestions have been advanced and can be grouped into two categories: suggestions that assume a local temperature rise in shear bands resulting from deformation [46] or/and suggestions that assume an increase of atomic mobility in the shear bands due to the increase of free volume within such localized regions [30,47]. However, none of these approaches could conclusively explained the opposite trends of nanocrystallization behavior observed in tensile and compression regions. Furthermore, since a similar temperature increase could be expected in compression or tension, the suggestion of temperature spike as the cause of crystallization may be rapidly excluded. Our finding and other published experimental results showed that the mechanically induced nanocrystallization is a general physical phenomenon, since it has been observed in many different amorphous alloy compositions such as Al-, Zr-, Pd and Fe-based alloys. Also, nanocrystallization occurs preferentially in the predominantly compressive region of deformed sample and may depend on the extent of plastic deformation. Jiang et al [48] have shown that ball milling for $1 \mathrm{~h}$ could form nanocrystallites in Al-based amorphous alloys, while ball milling, for $5 \mathrm{~h}$, could not cause the formation of nanocrystallites.

Based on the above and other experimental observations, the presence or absence of nanocrystallites during straining could be strongly linked with the microstructure configuration 
of shear bands in amorphous alloys. Indeed, based on nanovoids measurements inside shear bands during deformation [38, 49], it has been established a negative correlation between the nanovoids distribution and nanocrystallization development. In the compressive region where nanocrystallization is induced, a few residual nanovoids were observed in shear bands whereas in the tensile region, shear bands contain a higher density of nanovoids coupled with the absence of nanocrystals [38, 49]. Besides, Wright et al [50] have used the thermodynamics of nucleation to demonstrate that these nanovoids are predicted from dynamic evolution of highly unstable free volume in shear bands. During deformation of metallic glasses, the free volume in a shear bands is expected to increase; once a critical size is reached (of the order of tenths of Angstroms [50]) a driving force enhance voids nucleation via free volume coalescence. In tension, void growth and coarsen with the help of tensile stress state, leading to rapid shear bands propagation and premature fracture [51], whereas in compression, stress state (hydrostatic component) would hinder void formation.

Now, since the formation of nanocrystals within the narrow shear bands during deformation is attributed to local atomic mobility and diffusion inside shear bands [52], the above descriptions point out that the cause of the absence of nanocrystallization in shear bands at tension regions is that the excess free volume condenses rapidly into nanovoids during deformation, resulting in its reduced availability to enhance atomic mobility. In shear bands in which nanocrystallization exist, the available amount of excess free volume, which does not coalesce into nanovoids, can assist atomic transport, and thus makes nanocrystallization possible. Our analysis, hence, predict a transition of microstructure behaviour in shear band from nanocrystallization to voids nucleation depending on a critical size of accumulated free volume. This optimum critical size could be strongly affected by the intrinsic materials properties and the deformation conditions (i.e., stress state). 
With this background, one may conclude that the activated atomic mobility moderated by an optimum/critical amount of free volume is the main reason for mechanically induced crystallization. The opposite trends observed in tensile and compression regions can be rationalized as follow: during deformation, the free volume progressively increases in both compression and tension due to the flow dilatation [53, 54]. Once an optimum/critical volume size is reached, excess free volume rapidly condense into nanovoids and no crystallization could happen due to the reduced atomic diffusion rate; this is the case for the tensile stress state. In compression regions, stress state hamper excess free volume to reach a critical value and voids formation is retarded. Consequently, the available free volume governs atomic transport that could induce nanocrystallization, assisted by local shear stress. That explicitly, justifies the presence or absence of nanocrystallites in compression and tensile sides.

The present letter shows unambiguously that for describing the process of shear-induced nanocrystals formation inside the shear bands of metallic glasses, approaches based on assuming enhanced nucleation kinetics due to enhanced atomic mobility inside the shear bands are favorable. So, any proposed model should capture the principal feature of structural evolution in shear band described above. Crystallization process of amorphous alloy can be considered as a phenomenon describing via classic crystal nucleation and subsequent growth. The overall rate of transformation can be derived from the kinetic law based on Johnson-MehlAvrami (JMA) model [55]:

$\frac{\mathrm{d} \emptyset_{\mathrm{s}}}{\mathrm{dt}}=\mathrm{n}\left(k_{0} \exp \left(-\frac{\Delta \mathrm{G}^{\mathrm{c}}}{\mathrm{RT}}\right)\right)^{\mathrm{n}}\left(\frac{\gamma}{\dot{\gamma}}\right)^{\mathrm{n}-1} \exp \left(-\left(k_{0} \exp \left(-\frac{\Delta \mathrm{G}^{\mathrm{c}}}{\mathrm{RT}}\right) \frac{\gamma}{\dot{\gamma}}\right)^{\mathrm{n}}\right)$

Where $\theta_{\mathrm{s}}$ is the fraction of nucleated crystals, $\mathrm{k}_{0}$ is a material constant, $\mathrm{R}$ is gas constant and $\Delta \mathrm{G}^{\mathrm{c}}$ is the activation energy for overall crystallization process, $\mathrm{n}$ is Avrami exponent and $\mathrm{T}$ is 
the temperature $\dot{\gamma}$ is the total shear strain rate which can be decomposed into the elastic and plastic parts:

$\dot{\gamma}=\frac{\dot{\tau}}{\mu}+\dot{\gamma}^{\mathrm{p}}$

Where $\mu$ is the shear modulus. The homogeneous plastic flow is derived from the well-known equation of Speapen [53]:

$\dot{\gamma}^{\mathrm{p}}=2 \mathrm{f} \cdot \exp \left(-\frac{\delta \mathrm{v}^{*}}{\mathrm{v}_{\mathrm{f}}}\right) \exp \left(-\frac{\Delta \mathrm{G}^{\mathrm{m}}}{\mathrm{K}_{\mathrm{b}} \mathrm{T}}\right) \sinh \left(\frac{\tau \Omega}{2 \mathrm{~K}_{\mathrm{b}} \mathrm{T}}\right)$

where $\Delta \mathrm{G}^{\mathrm{m}}$ is the activation energy for an atomic jump, $\mathrm{f}$ is the jump frequency, $\tau$ is the applied shear stress, $\mathrm{k}_{\mathrm{B}}$ is the Boltzmann's constant, $\Omega$ is the atomic volume, $\delta$ is the geometrical factor of order $1, \mathrm{v}^{*}$ is the critical free volume and $\mathrm{v}_{\mathrm{f}}$ is the average free volume per atom. The dynamic evolution of free volume during straining under applied shear stress $\tau$ can be obtained from the free volume theory [56]. The net increase of the free volume is presented by the general equation:

$\frac{d v_{f}}{d t}=v^{*} \exp \left(-\frac{\delta v^{*}}{v_{f}}\right) \exp \left(-\frac{\Delta G^{m}}{K_{b} T}\right)\left\{\frac{2 \delta \cdot K_{b} \cdot T}{v_{f .} C_{e f f}}\left[\cosh \left(\frac{\tau \Omega}{2 K_{b} T}\right)-1\right]-\frac{1}{n_{D}}\right\}$

Where $\mathrm{n}_{\mathrm{D}}$ is the number of atomic jumps required to annihilate free volume, $\mathrm{C}_{\text {eff }}$ is the effective elastic modulus $\left(\mathrm{C}_{\text {eff }}=\mathrm{E} / 3(1-v), \mathrm{E}\right.$ is the Young's modulus and $v$ is the Poisson's ratio). The equations (3)-(6) provide a closed constitutive model for the shear induced-crystallization under an applied shear stress. These coupled non-linear equations are solved using fourth order Runge-Kutta scheme to highlight the variation of shear stress, free volume and subsequently 
the crystal volume fraction. The solution is, then, fitted to experimental data of figure 4(b) using an appropriate optimization algorithm. Figure 5 shows the experimentally obtained strain dependence of crystal volume fraction in the studied glassy alloy, together with the best-fit curve obtained on the basis of equations (3)-(6).The corresponding fitting parameters are summarized in table 1 . Figure 5 shows that the fitting route yields satisfactory results and the almost adjustable model parameters are physically meaningful and comparable to the literature. The figure shows that the crystalline phase does not transform all at once; the free volume content increases gradually and, after an incubation period, the crystallized volume fraction increases concurrently with the extent of deformation. The incubation period corresponds to the time for which the excess of free volume is sufficient to enhance atomic mobility helping nanocrystals nucleation. Figure 5 predicts also a saturation period for which the crystal fraction remains almost constant.

\section{Activation energy for nanocrystallization}

The activation energy is the most important parameter which governs crystallization rate because it represents the barrier energy for the phase transformation from an amorphous to a crystalline phase under plastic deformation. This means that as long as the activation energy is low, the nanocrystallization rate becomes faster, suggesting that additional mechanical energy can promote the precipitation of nanocrystallites. The value of activation energy for nanocrystallization reported in table 1 is close to $350 \mathrm{~kJ} / \mathrm{mol}$. This value remains lower than those reported in the literature for non-deformed Pd-based amorphous alloys $(452 \mathrm{~kJ} / \mathrm{mol}$ for $\mathrm{Pd}_{79} \mathrm{Cu}_{6} \mathrm{Si}_{10} \mathrm{P}_{5}$ [57] and $395 \mathrm{~kJ} / \mathrm{mol}$ for $\mathrm{Pd}_{76} \mathrm{~B}_{24}$ [58]) which are derived from Kissinger and Johnson-Mehl-Avrami (JMA) methods. The decrease of activation energy for crystallization is plausible and confirms that high deformation has an effect on the subsequent crystallization 
behavior. It can enhance atomic rearrangement and local fluctuation, favorable for easier nanocrystallization process with less required energy for atomic diffusion. The markedly decreasing of activation energy with the degree of deformation has been also confirmed in various $\mathrm{Cu}$-based glassy alloys. Ma et el [59] have experimentally found that with increasing degree of pre-deformation induced by cold-rolling, the crystallization activation energy for the CuZr-based BMG alloy decreases gradually. Wang et al [45] have recently established a negative relationship between the crystallized activation energy and the plastic strain of $\mathrm{CuZr}$ based bulk metallic glasses.

\section{Conclusion}

Deformation-induced nanocrystallization during bending has been quantitatively investigated in Pd-based metallic glass via a direct measurement of crystals volume fraction versus the extent of plastic deformation. The opposite trends of nanocrystallization behavior observed in tensile and compression regions has been reasonably explained by the negative correlation between the nanovoids distribution and nanocrystallization development in shear bands. A micromechanism-inspired constitutive model based on atomic structure in shear bands has been presented to capture the evolution of crystal volume fraction during straining. The effects of shear strain on the free volume and crystallization process have been accounted. The proposed model showed a good ability to reproduce the experimental data including several physical parameters, namely the activation energy for nanocrystallization.

\section{Acknowledgement}


The authors wish to dedicate this work to the memory of Professor Alain Reza Yavari who passed away and whom they sorely miss.

The authors would like to express their gratitude to King Khalid University, Saudi Arabia for providing administrative and technical support

\section{References}

[1] J. Eckert, J. Das, S. Pauly, C. Duhamel, Mechanical properties of bulk metallic glasses and composites, J. Mater. Res. 22 (2007) 285-301.

[2] M. Ashby, A.L. Greer, Metallic glasses as structural materials, Scr. Mater. 54 (2006) 321326

[3] C. A. Schuh, T. C. Hufnagel, and U. Ramamurty, Mechanical behavior of amorphous alloys, Acta Mater. 55 (2007) 4067.

[4] A. L. Greer, E. Ma, Bulk metallic glasses: at the cutting edge of metals research, MRS Bull. 32 (2007) 611.

[5] J. Das, M. B. Tang, K. B. Kim, R. Theissmann, F. Baier, W. H. Wang, J. Eckert, "Work-Hardenable" Ductile Bulk Metallic Glass Phys. Rev. Lett. 94 (2005) 205501.

[6] Y.J. Liu, H.W. Yao, T.W. Zhang, Z. Wang, Y.S. Wang, J.W. Qiao, H.J. Yang, Z.H. Wang, Designing ductile CuZr-based metallic glass matrix composites, Mater. Sci. Eng. A 682 (2017) $542-549$.

[7] R.F. Wu, Z.M. Jiao, Y.S. Wang, Z. Wang, Z.H. Wang, S.G. Ma, J.W. Qiao, Excellent plasticity of a new Ti-based metallic glass matrix composite upon dynamic loading, Mater. Sci. Eng. A 677 (2016) $376-383$.

[8] Y. Wu, D. Ma, Q.K. Li, A.D. Stoica, W.L. Song, H. Wang, X.J. Liu, G.M. Stoica, G.Y. Wang, K. An, X.L. Wang, M. Li, Z.P. Lu, Transformation-induced plasticity in bulk metallic glass composites evidenced by in-situ neutron diffraction, Acta Mater. 124 (2017) 478-488 
[9] J. Liu, Q. Wang, K. Sun, S. Gravier, J. Blandin, B. Sun, J. Liu, Serrated plastic flow behavior and microstructure in a Zr-based bulk metallic glass processed by surface mechanical attrition treatment, J. Iron Steel Res. Int. 24 (2017) 475-482

[10] R.T. Qu, Q.S. Zhang, Z.F. Zhang, Achieving macroscopic tensile plasticity of monolithic bulk metallic glass by surface treatment, Scr. Mater. 68 (2013) 845-848

[11] P. Xue, S. Pauly, W. Gan, S. Jiang, H. Fan, Z. Ning, Y. Huang, J. Sun, Enhanced tensile plasticity of a CuZr-based bulk metallic glass composite induced by ion irradiation, J. Mater. Sci. Technol. 35 (2019) 2221-2226

[12] J. Pan, Y.X. Wang, Y. Li, Ductile fracture in notched bulk metallic glasses, Acta Mater. 136 (2017) $126-133$

[13] W. Zhai, L. H. Nie, X. D. Hui, Y. Xiao, T. Wang, B. B. Wei, Ultrasonic excitation induced nanocrystallization and toughening of $\mathrm{Zr}_{46.75} \mathrm{Cu}_{46.75} \mathrm{Al}_{6.5}$ bulk metallic glass , J. Mater Sci \& Tech 45 (2020) 157-161.

[14] K. Hajlaoui, A. R. Yavari, B. Doisneau et al., Shear delocalization and crack blunting of a metallic glass containing nanoparticles: in situ deformation in TEM analysis, Scri. Mater. 54 (2006) 1829-1834.

[15] Z. Wang, K. Georgarakis, K. S. Nakayama, Y. Li, A. A. Tsarkov, G. Xie, D. Dudina, D. V. Louzguine-Luzgin, A. R. Yavari, Microstructure and mechanical behavior of metallic glass fiber-reinforced Al alloy matrix composites, Scientific Reports. 6 (2016) 24384

[16] M.K. Shete, I. Singh, R. Narasimhan, U. Ramamurty, Effect of strain hardening and volume fraction of crystal phase on strength and ductility of bulk metallic glass composites, Scr. Mater. 124 (2016) 51-55.

[17] T. Brink, M. Peterlechner, H. Rösne, K. Albe, G. Wilde, Influence of crystal nanoprecipitates on shear-band propagation in $\mathrm{Cu}-\mathrm{Zr}$ based metallic glasses, Phys. Rev. Appl. 5 (2016) 054005 
[18] Y. Wu, H. Wang, H. H. Wu, Z. Y. Zhang, X. D. Hui, G. L. Chen, D. Ma, X. L. Wang, and Z. P. Lu, Formation of $\mathrm{Cu}-\mathrm{Zr}-\mathrm{Al}$ bulk metallic glass composites with improved tensile properties, Acta Mater. 59 (2011) 2928

[19] D. C. Hofmann, J.-Y. Suh, A. Wiest, G. Duan, M.-L. Lind, M. D. Demetriou, and W. L. Johnson, Designing metallic glass matrix composites with high toughness and tensile ductility, Nature 451(2008) 1085

[20] S.F. Guo, L. Liu, N. Li, Y. Li, Fe-based bulk metallic glass matrix composite with large plasticity, Scri. Mater. 62 (2010) 329.

[21] Y. Chen J-Z Jiang, A novel strategy to design Zr-Co-Al BMG composites containing only B2-ZrCo phase, Intermetallics 123 (2020) 106821

[22] K. Hajlaoui, N. Alsaleh, N.H. Alrasheedi, A.R. Yavari, Coalescence and subsequent twinning of nanocrystals during deformation of CuZr-based metallic glasses: The grain size effect, J. of Non-Crys. Sol. 464 (2017) 39-43.

[23] K. Hajlaoui, N.H. Alrasheedi, A.R. Yavari, Direct observation of shear-induced nanocrystal attachment and coalescence in CuZr-based metallic glasses: TEM investigation, J.Alloys and Comp. 665 (2016) 339-344.

[24] S. Pauly, G. Liu, S. Gorantla, G.Wang, U. Kuhn, D.H. Kim, J. Eckert, Criteria for tensile plasticity in $\mathrm{Cu}-\mathrm{Zr}-\mathrm{Al}$ bulk metallic glasses, Acta Mater. 58 (2010) 4883-4890.

[25] S. Pauly, S. Gorantla, G.Wang, U. Kühn, J. Eckert, Transformation-mediated ductility in CuZr-based bulk metallic glasses, Nat. Mater. 9 (2010) 473-477

[26] H. Chen, Y. He, G.J. Shiflet, SJ Poon., Deformation-induced nanocrystal formation in shear bands of amorphous alloys, Nature 367 (1994) p.541

[27] H.J. Jin, F. Zhou, L.B. Wang, and K. Lu, Effect of plastic deformation on thermal stability in metallic glasses. Scripta Mater. 44 (2001) 1083 
[28] G.J. Fan, M.X. Quan, Z.Q. Hu, W. Löser, and J. Eckert: Deformation- induced microstructural changes in Fe40Ni40P14B6 metallic glass, J. Mater. Res. 14 (1999) 3765.

[29] S.H. Joo, D.H. Pi, A. D. H. Setyawan, H.ㅁato, M.Janecek, Y.C. Kim, S.Lee, H.Skim, Work-Hardening Induced Tensile Ductility of Bulk Metallic Glasses via High-Pressure Torsion, Scientific Reports, 5 (2015) 9660.

[30] J.J. Kim, Y. Choi, S. Suresh, and A.S. Argon: Nanocrystallization during nanoindentation of a bulk amorphous metal alloy at room temperature. Science 295 (2002) 654

[31] S.W. Lee, M.Y. Huh, S.W. Chae, J.C. Lee, Mechanism of the Deformation-Induced Nanocrystallization in a Cu-Based Bulk Amorphous Alloy Under Uniaxial Compression, Scr. Mater. 54 (2006) 1439-1444.[32] A. Hirata, P. Guan, T. Fujita, Y. Hirotsu, A. Inoue, A.R. Yavari, T. Sakurai, M. Chen, Direct observation of local atomic order in a metallic glass, Nat. Mater. 10 (2011) 28-33.

[33] A. R. Yavari, K. Georgarakis,J. Antonowicz, M. Stoica, N. Nishiyama, G. Vaughan, M. Chen, M. Pons, Crystallization during Bending of a Pd-Based Metallic Glass Detected by XRay Microscopy, Phy. Rev. Lett. 109 (2012) 085501.

[34] Z. Suo, E. Y. Ma, H. Gleskova, and S. Wagner, Mechanics of rollable and foldable filmon-foil electronics, App. Phys. Lett. 74 (1999) 1177.

[35] M. Aljerf, K. Georgarakis, A.R. Yavari, Shaping of metallic glasses by stress-annealing without thermal embrittlement, Acta Materialia, 59 (2011) 3817-3824.

[36] http://www.esrf.eu/UsersAndScience/Experiments/StructMaterials/ID11.

[37] K. Wang, T. Fujita, Y.Q. Zeng, N. Nishiyama, A. Inoue, M.W. Chen, Micromechanisms of serrated flow in a $\mathrm{Ni}_{50} \mathrm{Pd}_{30} \mathrm{P}_{20}$ bulk metallic glass with a large compression plasticity, Acta Materialia 56 (2008) 2834-2842. 
[38] W.H. Jiang, M. Atzmon, The effect of compression and tension on shear-bandstructure and nanocrystallization in amorphous $\mathrm{Al}_{90} \mathrm{Fe}_{5} \mathrm{Gd}_{5}$ : a high-resolution transmission electron microscopy study, Acta Materialia 51 (2003) 4095-4105.

[39] Z.J. Yan, Y. Hu, K.K. Song, F.P. Dai, J. He, J. Eckert, Vickers-indentation-induced crystallization in a metallic glass, Appl. Phys. Lett. 106 (2015) 101909.

[40] Z. Ma, F. Lu, X. Ma, H. Zhao, K. Zeng, L. Ren, Cyclic stress induced surface nanocrystallization adjacent to indentation edge of Zr-based bulk metallic glass at room temperature, Appl. Surface Sci. 506 (2020) 145044.

[41] Ch. Ma, N. Nishiyama, A. Inoue, Phase Equilibria and Thermal Stability of Pd-Cu-Ni-P Alloys, Materials Transactions, 43 (2002) 1161-1165.

[42] P. Wesseling, B.C. Ko, J.J. Lewandowski, Quantitative evaluation of a-Al nano-particles in amorphous $\mathrm{Al}_{87} \mathrm{Ni}_{7} \mathrm{Gd}_{6}$-comparison of XRD, DSC, and TEM, Scri.Mater. 48 (2003) 15371541.

[43] T. Gloriant, M. Gich, S. Surinach, M.D. Baro, A.L. Greer, Evaluation of the volume fraction crystallized during devetrification of Al-based amorphous alloy, J. Metastable Nanocryst. Mater. 8 (2000) 365- 370.

[44] F. Qiu, P. Shen, T. Liu, Q. Jiang, Enhanced ductility in a $\mathrm{Zr}_{65} \mathrm{Cu}_{15} \mathrm{Al}_{10} \mathrm{Ni}_{10}$ bulk metallic glass by nanocrystallization during compression, Materials and Design 36 (2012) 168-171 [45] T. Wang, Y. Wu, J. Si, Y. Liu, X. Hui, Plasticizing and work hardening in phase separated $\mathrm{Cu}-\mathrm{Zr}$-Al-Nb bulk metallic glasses by deformation induced nanocrystallization, Materials \& Design. 142 ( 2018) 74-82

[46] J. J. Lewandowski, A. L. Greer, Temperature rise at shear bands in metallic glasses, Nature Mater. $5(2006) 15$. 
[47] R. J. Hebert, J. H. Perepezko, H. Rösner, G. Wilde, Dislocation formation during deformation-induced synthesis of nanocrystals in amorphous and partially crystalline amorphous $\mathrm{Al}_{88} \mathrm{Y}_{7} \mathrm{Fe}_{5}$ alloy, Scr. Mater. 54 (2006) 25-29.

[48] W.H. Jiang, F.E. Pinkerton, M. Atzmon, Deformation-induced nanocrystallization: A comparison of two amorphous Al-based alloys, J. Mater. Res. 20 (2005) 696-702.

[49] W.H. Jiang A, M. Atzmon, Mechanically-Assisted Nanocrystallization And Defects In Amorphous Alloys: A High-Resolution Transmission Electron Microscopy Study Scr. Mater. 54 (2006) 333-336

[50] W. J. Wright, T. C. Hufnagel, W. D. Nix, Free Volume Coalescence And Void Formation In Shear Bands In Metallic Glass, J.App. Phy. 93 (2003) 1432- 1437

[51] H.J. Chang, S.Y. Kim, W.-J. Moon, Y.-M. Kim, E.S. Park, D.H. Kim, Probing structural changes during ductile fracture in metallic glasses via in situ straining inside a $\mathrm{MeV}$ transmission electron microscope, Intermetallics 102 (2018) 94-100.

[52] J. Bokeloh, S. V. Divinski, G. Reglitz, and G. Wilde, Tracer measurements of atomic diffusion inside shear bands of a bulk metallic glass, Phys. Rev. Lett. 107 (2011) 235503

[53] F. Spaepen, A microscopic mechanism for steady state inhomogeneous flow in metallic glasses, Acta. Metall. 25 (1976) 407.

[54] A. S. Argon, Plastic deformation in metallic glasses. Acta Metall, 27 (1979) 47-58.

[55] M. Avrami, "Kinetics of phase change. II Transformation-time relations for random distribution of nuclei," The Journal of Chemical Physics. 8 (1940) 212-224.

[56] M.H. Cohen, D. Turnbull, Molecular transport in liquids and glasses. The Journal of Chemical Physics.31 (1959) 1164-1169

[57] L. Liu, X. Zhao, Ch. Ma, T. Zhang, Kinetics of crystallization process for Pd-based bulk metallic glasses, Intermetallics 17 (2009) 241-245 
[58] A Lucci, G Meneghini, activation energy for nucleation and crystal growth during the crystallization of palladium-based metallic glasses containing boron and beryllium, Thermochrmlca Acta, 71 (1983) 147-159

[59] G. Z. Ma, K. K. Song, B. A. Sun, Z. J. Yan, U. Ku“hn, D. Chen, J. Eckert, Effect of coldrolling on the crystallization behavior of a CuZr-based bulk metallic glass, J Mater Sci 48 (2013) 6825-6832. 


\section{Tables :}

\begin{tabular}{lc}
\hline Parameters & Values \\
\hline $\mathrm{f}\left(\mathrm{s}^{-1)}\right.$ & $10^{13}$ \\
\hline$\delta$ & 0.15 \\
\hline$\Delta \mathrm{G}^{\mathrm{m}}(\mathrm{J})$ & $10^{-19}$ \\
\hline $\mathbf{K}_{\mathbf{b}}\left(10^{-24} \mathrm{~J} / \mathrm{K}\right)$ & 13.8 \\
\hline $\mathrm{T}(\mathrm{K})$ & 298 \\
\hline $\mathbf{\Omega}\left(10^{-29} \mathrm{~m}^{3}\right)$ & 1.64 \\
\hline$v$ & 0.39 \\
\hline$\mu(\mathrm{GPa})$ & 33 \\
\hline $\mathrm{n}_{\mathrm{D}}$ & 3 \\
\hline $\mathrm{k}_{0}$ & 3.64 \\
\hline$\Delta \mathrm{G}^{\mathrm{c}}(\mathrm{kJ} / \mathrm{mol})$ & 350 \\
\hline $\mathrm{n}$ & \\
\hline $\mathrm{R}(\mathrm{J} / \mathrm{mol} \mathrm{K})$ & \\
\hline
\end{tabular}

Table 1. Fitting parameters obtained from simulation of crystal volume fraction during deformation. 


\section{Figure Captions}

Figure 1. (a) SEM micrograph in the center of the bent $\mathrm{Pd}_{40} \mathrm{Cu}_{30} \mathrm{Ni}_{10} \mathrm{P}_{20}$ metallic glass ribbon: both tensile and compressive sides, (b) graphic illustration of the curvature radius, R, distance $\mathrm{y}$ and the strains within a ribbon subjected to a bending moment.

Figure 2. Diffraction pattern taken on both sides of the neutral axis of the bent $\mathrm{Pd}_{40} \mathrm{Cu}_{30} \mathrm{Ni}_{10} \mathrm{P}_{20}$ glassy ribbon using synchrotron light in transmission. Detection of very fine rings in the compressed side indicating the presence of nanocrystals.

Figure 3: Synchrotron XRD diffraction profiling of deformed area of bent ribbon; the annotations $\mathrm{D}_{0}, \mathrm{D}_{4}, \mathrm{D}_{8} \ldots \ldots . . \mathrm{D}_{20}$, correspond to the steps during scanning of the deformed zones with the X-ray beam.

Figure 4: a) The relative areas of the deconvoluted amorphous and crystalline peaks in XRD diffraction pattern, (b) nanocrystal volume fraction versus strain determined from XRD analyses.

Figure 5. Crystal volume fraction versus stain deformation of Pd-based metallic glass. The dash curve is obtained from the best fit to equations (3)-(6). 


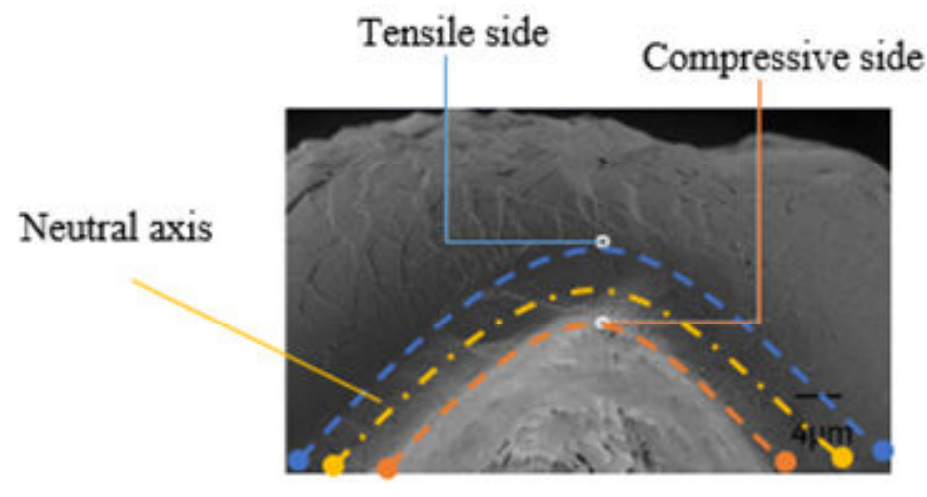

(a)

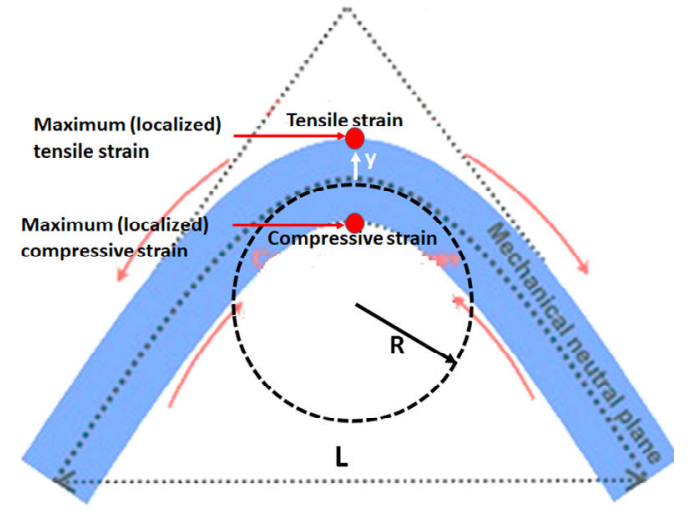

(b)

Figure 1 


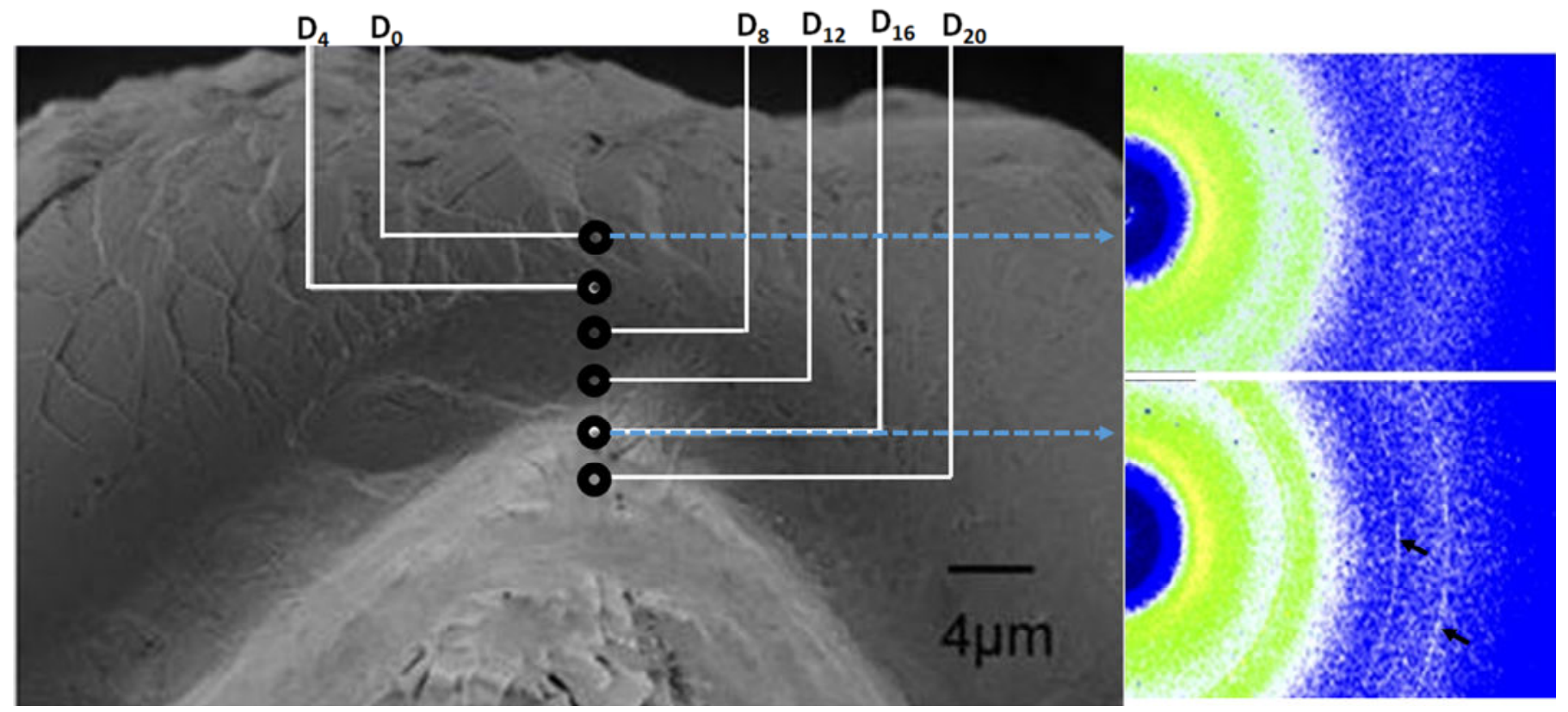

Figure 2 


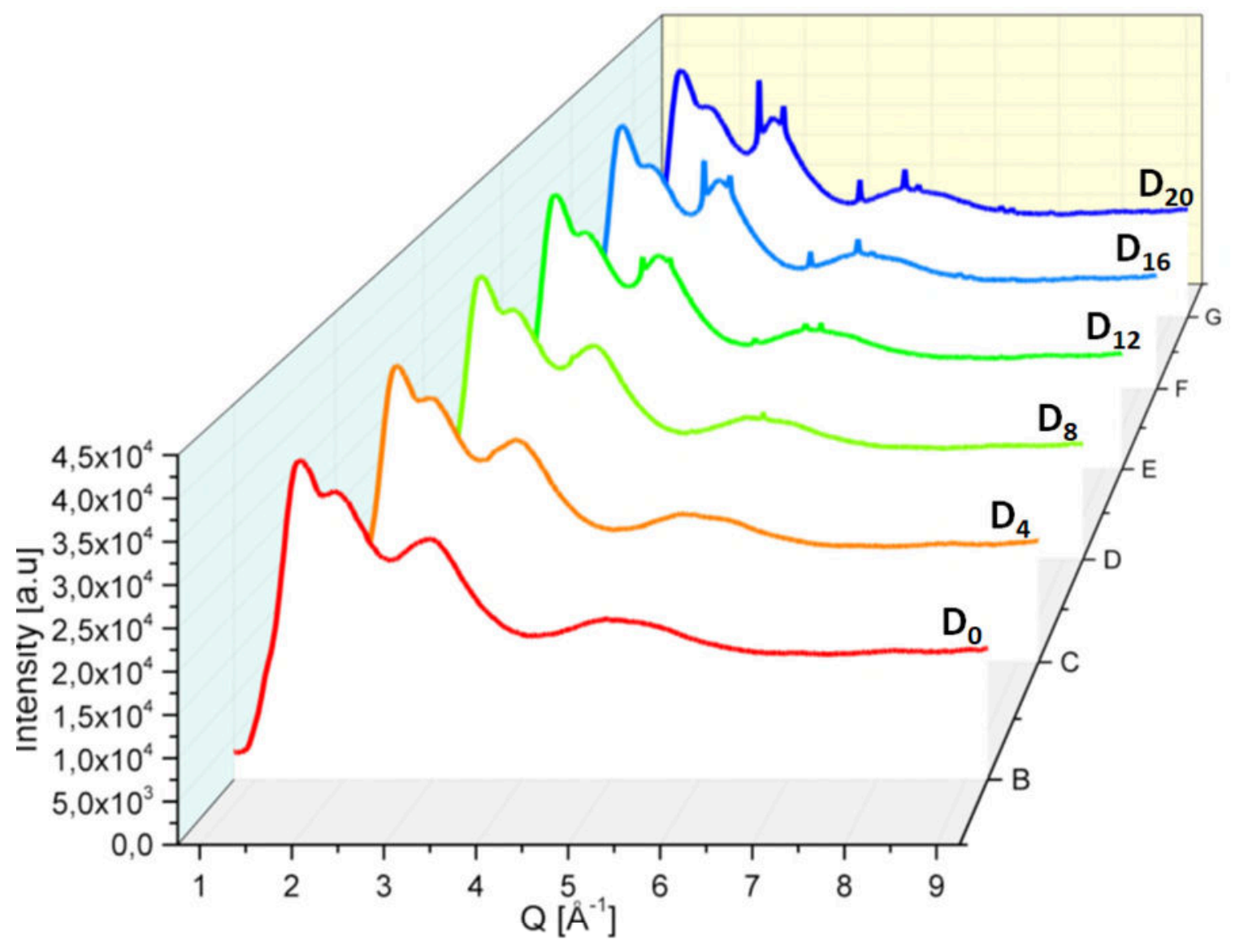

Figure 3 


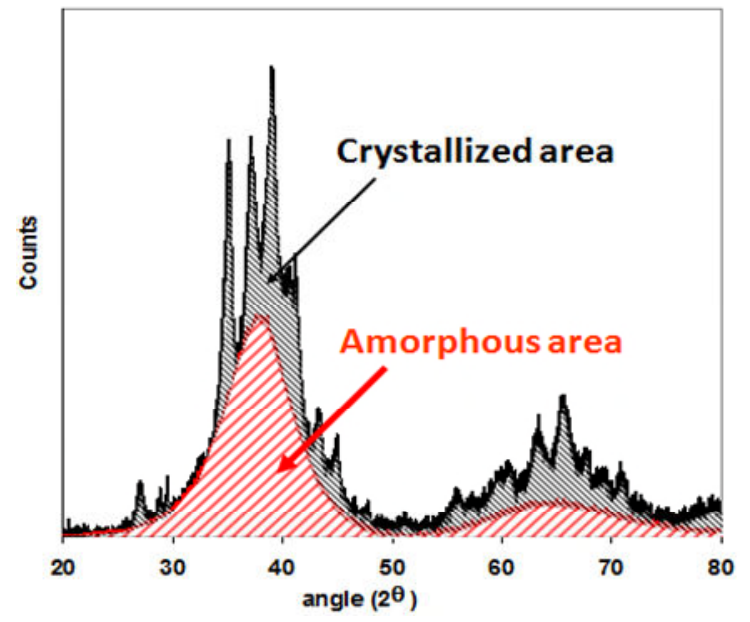

(a)

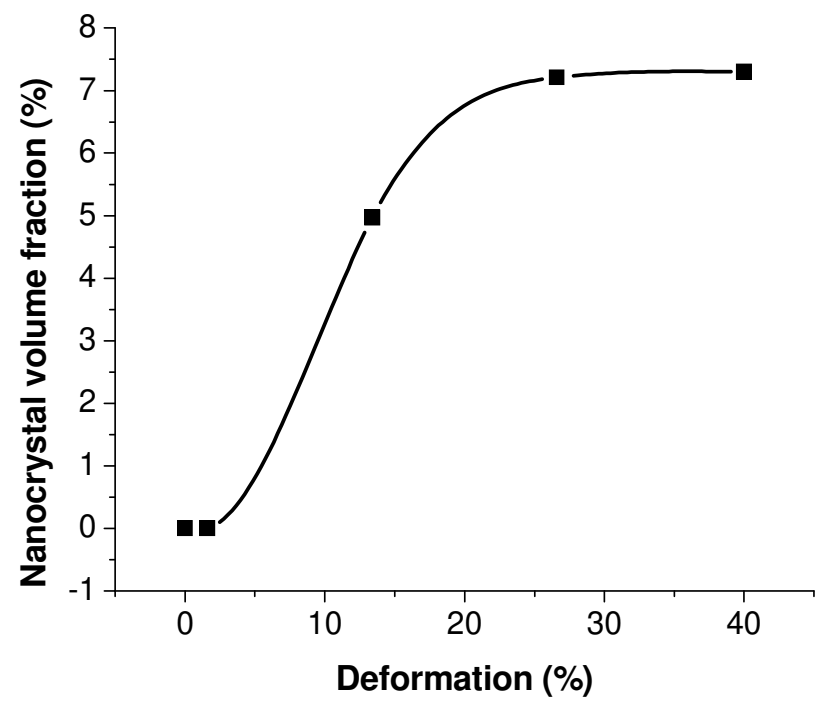

(b)

Figure 4 


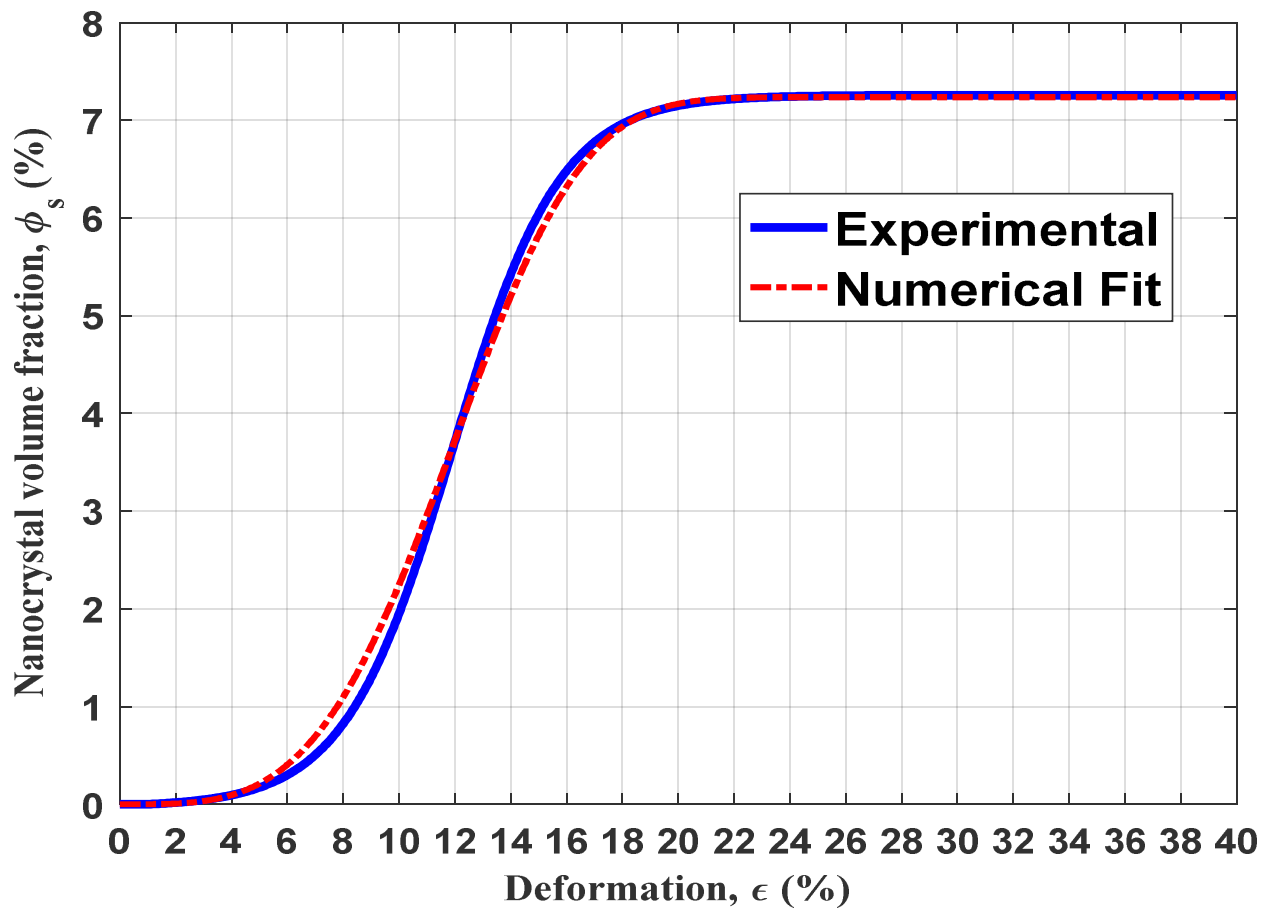

Figure 5 
$2020-11-10$

\title{
Investigation on the
}

mechanically-induced

\section{nanocrystallization in metallic glasses}

\author{
Ammari, C.
}

Elsevier

\author{
Ammari C, Yousfi MA, Hajlaoui K, Georgarakis K. (2021) Investigation on the \\ mechanically-induced nanocrystallization in metallic glasses. Journal of Alloys and Compounds, \\ Volume 859, April 2021, Article number 157864 \\ https://doi.org/10.1016/j.jallcom.2020.157864 \\ Downloaded from Cranfield Library Services E-Repository
}

